\title{
L'Amérique centrale 1979-1990: de l'internationalisation a la régionalisation des crises locales
}

\section{Georges Couffignal}

\section{(2) OpenEdition \\ Journals}

Édition électronique

URL : http://journals.openedition.org/conflits/523

DOI : $10.4000 /$ conflits.523

ISSN : $1777-5345$

Éditeur :

CCLS - Centre d'études sur les conflits lilberté et sécurité, L'Harmattan

Édition imprimée

Date de publication : 6 décembre 1992

ISSN : 1157-996X

Référence électronique

Georges Couffignal, «L'Amérique centrale 1979-1990 : de l'internationalisation a la régionalisation des crises locales », Cultures \& Conflits [En ligne], 08 | hiver 1992, mis en ligne le 27 janvier 2003, consulté le 30 mars 2021. URL : http://journals.openedition.org/conflits/523 ; DOI : https://doi.org/10.4000/ conflits.523

Ce document a été généré automatiquement le 30 mars 2021.

Creative Commons License 


\title{
L'Amérique centrale 1979-1990 : de l'internationalisation a la régionalisation des crises locales
}

\author{
Georges Couffignal
}

De 1979 à 1990 l'Amérique Centrale, région oubliée car traditionnelle "arrière-cour" ("backyard") des Etats-Unis, a occupé progressivement le devant de la scène internationale et a focalisé l'attention de l'opinion publique mondiale. Elle est depuis cette date retournée dans le relatif désintérêt qui la caractérisait auparavant. Durant cette période, à l'exception du Belize (ancien Honduras britannique, qui accède sereinement à l'indépendance en 1981), les pays qui la composent ont connu des tensions sans précédents, se sont transformés en profondeur, ont servi de laboratoire à toute une série de changements politiques, à l'intérieur des frontières ou hors d'elles ${ }^{1}$. A l'intérieur tout d'abord, sous l'effet de l'internationalisation des conflits nicaraguayen et salvadorien, voulue par les Etats-Unis, tous les Etats de la région ont connu des processus parallèles de militarisation et de démocratisation, alors même que dans le passé la militarisation avait en général été synonyme de négation de la démocratie. Hors des frontières en second lieu, plusieurs Etats proches des zones de conflits, craignant que cette proximité ne les entraîne à leur tour dans des conflits ou n'apporte un cortège de problèmes sur leur propre territoire, ne ménagèrent pas leurs efforts en vue de trouver une réponse régionale à ces crises. La conséquence fut d'une part l'émergence de nouveaux acteurs, d'autre part la naissance d'une ébauche de diplomatie latino-américaine autonome. Ces mutations de la scène inter-américaine auraient pu n'être que conjoncturelles et passagères. Mais la disparition de l'affrontement bipolaire en fin de période a eu pour effet de les pérenniser.

Démocratisation et militarisation des régimes, conséquence de l'internationalisation des conflits

Deux pays symbolisent les transformations que va connaître la région, deux pays que l'on trouve curieusement au début et à la fin de la période. Le premier aux marges de l'Amérique centrale, le second en son centre, à savoir le Panama et le Nicaragua. 
En 1968, un général progressiste, Omar Torrijos, avait pris le pouvoir à Panama. Nationaliste avant tout, il cherchait à conquérir pour son pays une marge d'autonomie vis-à-vis des Etats-Unis, et n'hésitait pas pour ce faire, à afficher de bonnes relations avec Cuba. L'élection de Jimmy Carter à la présidence des USA, le cours nouveau qu'il cherche à donner aux relations Etats-Unis-Amérique latine, en application des recommandations de la Trilatérale (rapport Sol-Linowitz), vont lui permettre de négocier des traités prévoyant la restitution de la souveraineté panaméenne sur le canal le 31 décembre 1999. Cette restitution devait être préparée, en particulier par un transfert progressif des responsabilités ${ }^{2}$. La signature de ces traités, en 1977, eut un impact considérable en Amérique centrale. Pour la première fois dans l'histoire de la région, il semblait possible qu'un petit pays puisse affirmer son droit à l'autonomie face aux Etats-Unis, sans que cela provoque de réactions négatives de leur part..

Parallèlement, Jimmy Carter développait son discours de défense des droits de l'homme, et le concrétisait en réduisant ou suspendant l'aide américaine au Salvador et au Guatemala, en raison des atteintes aux droits qui y étaient perpétrées. Le résultat en fut la polarisation croissante de ces pays, entre une classe restreinte de grands possédants accrochés à leurs privilèges, et des masses de paysans qui ne voyaient aucune perspective de débouché politique à leur aspiration au mieux-être. Les sinistres "escadrons de la mort" apparurent au Salvador et se firent une spécialité de l'assassinat d'opposants, de responsables syndicaux ou associatifs, de journalistes, voire de gens d'Eglise, comme l'archevêque de San Salvador, Monseigneur Romero, abattu dans sa cathédrale, pendant qu'il officiait. Le processus est identique au Guatemala, en y ajoutant une composante ethnocide. L'armée multiplie les exactions contre les groupes indiens, n'hésitant pas à massacrer tous les habitants de certains villages, au motif rhétorique d'éradiquer la guérilla. L'absence de perspective politique favorise en effet dans ces deux pays le développement de mouvements d'opposition qui n'ont d'autres moyens d'expression politique que la lutte armée.

Ces mouvements de guérillas sont d'autant plus convaincus de la faisabilité de leur projet qu'au Nicaragua, et c'est le second fait majeur du début de la période, le Front Sandiniste de Libération Nationale (FSLN) renverse en juillet 1979 le régime dictatorial de Anastasio Somoza. Outre l'extraordinaire légitimité que confère la victoire du FSLN à la lutte armée, chacun note l'attitude des Etats-Unis, conséquente avec la nouvelle orientation de la politique américaine voulue par J. Carter. Bien que la dynastie Somoza ait été mise en place en 1934 par les USA, ceux-ci commencent par couper l'aide militaire et économique (février 1978) à un pays qui est un de leurs plus anciens alliés dans la région, puis ils ne s'opposent pas au renversement du dictateur (juillet 1979) et reconnaissent immédiatement le nouveau régime, bien plus, ils lui accordent d'entrée 16 millions de dollars d'aide, qui atteignent 75 millions en $1980^{3}$.

Ces deux faits - traités Carter-Torrijos en 1977 et victoire sandiniste en 1979 - sont à l'origine de toutes les tensions et transformations que va connaître la région dans les années 1980. Deux faits où les Etats-Unis ont fait montre d'une souplesse et d'une compréhension que dénonce avec virulence l'ancien gouverneur de l'Etat de Californie, Ronald Reagan, qui se présente contre Jimmy Carter aux élections de 1981, et le bat.

Prenant le contre-pied de son prédécesseur, le nouveau président soutient que les USA ont baissé la garde face à l'Union Soviétique. Il va chercher à mobiliser le maximum de ressources dans sa croisade contre "l'empire du mal". Dès 1981, le budget militaire passe de 24 à $29 \%$ des dépenses fédérales. Il faut restaurer ce qu'il appelle une marge de 
sécurité vis-à-vis de l'Union Soviétique et la contraindre à reculer partout où elle a marqué des points, notamment en Afghanistan et en Amérique centrale. Pour R. Reagan, la victoire du front sandiniste au Nicaragua, l'implantation du Front Farabundo Marti de Liberation Nationale (FMLN) au Salvador (lequel a lancé une "offensive finale" infructueuse à la veille de sa prise de fonctions), ne s'expliquent que dans le cadre de la compétition Est-Ouest. Il convient d'endiguer ("containment") l'avancée du communisme. Il faut donc empêcher de vaincre ceux qui, dit-il, lui sont subordonnés, comme le FMLN. Il faut aussi et surtout l'obliger à reculer ("roll-back") là où il a pris des positions, comme au Nicaragua.

La suite logique est une militarisation croissante de tous les pays de la région ${ }^{4}$. Les budgets militaires gonflent démesurément, en partie grâce aux fonds reçus au titre de l'aide au développement en provenance des Etats-Unis, aide qui augmente de manière spectaculaire ${ }^{5}$. Au Nicaragua, le pluralisme du début s'éloigne, ce qui conforte la stratégie nord américaine. Dès 1981, on avait commencé à voir les éléments modérés de la coalition victorieuse se séparer d'un pouvoir aux orientations trop radicales. Très vite, les Etats-Unis vont activement soutenir une insurrection contre-révolutionnaire, la "Contra" (les "combattants de la liberté" pour R. Reagan). Elle est formée au début par d'anciens gardes somozistes, rejoints ensuite par des paysans fuyant la conscription obligatoire, voire par des sandinistes de la première heure déçus par l'évolution et la radicalisation du régime. Ces groupes armés ont leurs bases à l'extérieur du pays, au Costa Rica et surtout au Honduras. Malgré sa tradition de neutralité, le Costa Rica se trouve ainsi engagé dans la spirale de la militarisation ${ }^{6}$, tandis que le Honduras devient un immense champ de bases militaires. Les Honduriens en viennent à qualifier leur capitale, Tegucigalpa, de "Saïgoncito", petit Saïgon. L'image est claire. Il est vrai que dans ce pays opèrent, outre l'armée régulière, les troupes de la "Contra", celles des Etats-Unis, qui développent leurs bases et agrandissent les pistes d'atterrissage afin qu'elles puissent recevoir des gros porteurs en cas d'intervention au Nicaragua, enfin celles du FMLN dans les camps de réfugiés à la frontière avec le Salvador.

Dans ce dernier pays, la guerre civile s'exacerbe. Tant le gouvernement que la guérilla voient leurs soutiens respectifs se renforcer: Managua et Cuba pour le FMLN, Washington pour le gouvernement. Au total, le Honduras et le Salvador vont absorber près de 70\% de l'aide accordée par les Etats-Unis à l'Amérique centrale de 1977 à 1989, celle-ci connaissant une croissance spectaculaire et passant d'un peu moins de 200 millions de dollars en 1980 à près de 4 milliards de dollars de 1985 à 1989. Ces chiffres, il est vrai, concernent à la fois l'aide militaire et l'aide économique. Mais on sait qu'une part non négligeable de cette dernière fut détournée à des fins militaires. Quant au Nicaragua, plus de 50\% de son budget est à partir de 1985 consacré à l'effort de guerre. Les militaires, on le voit, occupent une place de plus en plus prépondérante dans les Etats de la région.

Parallèlement à cette militarisation, les Etats-Unis promeuvent une nouvelle stratégie : la "guerre de basse intensité" ("Low Intensity Warfare"). Le Pentagone a tiré les leçons de l'aventure vietnamienne. Face à un mouvement armé qui plonge ses racines dans un projet de libération nationale, le seul emploi massif de troupes et de moyens matériels sophistiques est impuissant à réduire l'adversaire. L'exécutif va donc s'efforcer, tout en soutenant les opérations militaires classiques menées par la "Contra", d'agir sur deux terrains. Il faut tout d'abord obtenir l'appui constant de l'opinion publique nord américaine. L'administration Reagan ne ménagera donc pas ses efforts en direction du Congrès, lequel est d'ailleurs à majorité républicaine durant le premier mandat ${ }^{7}$. Il faut 
de plus agir sur les élites du ou des pays théâtres du conflit, et d'une manière générale sur l'ensemble de la société civile concernée. L'objectif ici est de faire perdre toute légitimité à l'ennemi. Cette bataille est conçue comme aussi importante, sinon plus, que la bataille militaire. Dès lors, sur le terrain, la distinction personnel civil/personnel militaire devient floue : les uns comme les autres doivent être à même de réaliser des opérations très diverses, (économiques psychologiques... ), susceptibles d'aider à la réalisation de l'objectif contre-révolutionnaire ${ }^{8}$.

C'est pour gagner cette bataille idéologique que les Etats-Unis, et ce n'est pas le moindre des paradoxes de la période, vont pousser les militaires au pouvoir en Amérique centrale à laisser la place à des civils. En 1975, rappelons-le, seul le Costa Rica était doté d'un régime politique pluraliste, de type présidentiel. Partout ailleurs sévissaient des régimes autoritaires ou dictatoriaux, où le pouvoir était accaparé par un homme ou par une caste militaire. Les Etats-Unis, qui jusqu'alors toléraient, soutenaient ou avaient parfois suscité ces régimes au nom de la lutte contre la subversion, oeuvrent désormais pour le retour à des régimes représentatifs. Il s'agissait pour eux de démontrer la véracité du célèbre théorème énoncé par leur ambassadrice à l'ONU, Jane Kirckpatrik, selon lequel il était possible de sortir des dictatures dans le monde capitaliste mais impossible dans le monde communiste. L'objectif était d'isoler le Nicaragua sur la scène internationale, en montrant que les dirigeants de ce pays refusaient de se soumettre à la sanction d'élections pluralistes, alors qu'ils étaient entourés de pays où les gouvernants étaient désignés par le suffrage universel. Le discours de la démocratie était au début purement formel, à destination de l'opinion publique nord-américaine. A la fin de la décade, il était pourtant "devenu la force motrice de la politique nord-américaine dans l'hémisphère" ${ }^{19}$

C'est durant cette période que les notions de légalité et de légitimité sont introduites dans ces républiques où la notion d'Etat de droit n'avait pas grande signification, républiques dont l'histoire était avant tout celle d'une succession ininterrompue de coups d'Etats et de régimes plus ou moins répressifs. De nouvelles constitutions sont élaborées, des présidents sont élus au suffrage universel en 1981 au Honduras, 1984 au Salvador, 1985 au Guatemala. Le phénomène est concomitant, on le sait, dans les dictatures d'Amérique du Sud (Uruguay, Argentine, Brésil), qui tombent les unes après les autres avec la bénédiction des Etats-Unis (seuls le Chili et le Paraguay attendront la fin de la décennie pour recouvrer des régimes civils). Le Nicaragua enfin, soucieux de démontrer l'inanité des accusations de Ronald Reagan, se dote lui aussi d'une constitution et Daniel Ortega, "comandante" de la Révolution, est aisément élu président en 1984, une partie de l'opposition ayant il est vrai boycotté un scrutin que Washington qualifiera de "farce" malgré l'opinion positive de la plupart des observateurs internationaux sur leur déroulement ${ }^{10}$. Six ans plus tard, les sandinistes perdront les élections et abandonneront le pouvoir avec amertume mais finalement sans provoquer de troubles. Le résultat paradoxal de l'internationalisation voulue par R. Reagan a donc été, on le voit, de favoriser le retour à des régimes constitutionnels dans la plupart des pays de la zone. Seul Panama, au moment de la défaite sandiniste en février 1990, faisait encore exception.

Ce retour est probablement durable. Certes, dans nombre de pays, l'armée a conservé une place prépondérante. C'est la cas au Guatemala, où l'espace du pouvoir civil est très réduit, c'est le cas au Salvador, où l'armée se cache derrière l'ARENA, formation d'extrême-droite qui gouverne depuis 1989. C'est le cas enfin du Nicaragua, où l'armée est demeurée sous le contrôle des sandinistes après leur défaite électorale, ceci en 
accord avec la présidente Violeta Chamorro, afin d'assurer une alternance pacifique ${ }^{11}$. Mais cette situation n'est probablement pas durable à terme. En effet la fin en $1990 \mathrm{du}$ conflit nicaraguayen, suivie en 1992 de celle du conflit salvadorien, ont pacifié la zone. Les Etats-Unis n'ayant plus rien à redouter d'une Union Soviétique qui a disparu, et la survie du régime cubain étant elle-même fortement compromise, Washington devrait avoir à coeur de maintenir l'image démocratique acquise par les Etats centraméricains. Il est donc probable qu'ils freineront toute tentation, certainement très forte au Guatemala et au Salvador, des militaires d'occuper à nouveau le devant de la scène. Il est donc permis de parier sur le long terme. L'existence de partis, légalement constitués et de processus politiques compétitifs ${ }^{12}$ ne peuvent qu'amener à une transformation profonde de ces Etats, dans le sens d'une plus grande démocratie.

Quelle forme prendra-t-elle? Il est difficile de le prévoir. A l'instar des autres pays d'Amérique Latine, ceux d'Amérique centrale sont en effet confrontés au défi redoutable d'avoir à implanter une culture de démocratie dans une population qui en est dépourvue, ceci au moment où cette population doit affronter de graves situations de paupérisation. Outre les problèmes, classiques serait-on tenté de dire, de gestion de la dette et de transformation du modèle de développement, avec leur cortège d'austérité, de réduction des déficits budgétaires, d'abandon des politiques sociales, les Etats d'Amérique centrale sont véritablement sinistrés par les effets des conflits qui les ont ensanglantés. Pays agricoles pour la plupart, ils ont vu leurs productions chuter, les populations être déplacées, les Etats-Unis mesurer chichement leur aide dès que le spectre "communiste" s'est éloigné. Pour beaucoup de pays, à commencer par le Nicaragua, la crise économique s'accompagne d'immenses besoins de reconstitution des structures de base de l'économie. Le danger est redoutable d'un désintérêt ou d'un rejet de la démocratie non pas en provenance des oligarchies, comme dans le passé, mais en provenance des couches populaires de ces pays. Pourquoi voter quand aucune perspective d'amélioration économique ne se profile et que les canaux de représentation passent traditionnellement par des structures groupales (clientèlaires ou communautaires) beaucoup plus que par l'élection ${ }^{13}$ ? L'économie est en tout cas le thème premier des réunions désormais régulières des présidents centraméricains. Après avoir initié la formule de ces rencontres dans les années 1980 pour résoudre des questions avant tout politiques, ils se préoccupent désormais essentiellement de problèmes économiques. Ces rencontres elles-mêmes méritent d'être analysées. Elles sont le fruit de ce processus de régionalisation des conflits qui a initié un nouveau cours dans les relations interaméricaines.

Régionalisation des conflits, conséquence de l'émergence de nouveaux acteurs

L'un des multiples effets inattendus de la position volonté d'internationalisation de Ronald Reagan a paradoxalement été la naissance d'une diplomatie autonome et concertée de la part de plusieurs pays d'Amérique latine et d'Amérique centrale. Cinq dates rythment cette réaction à l'internationalisation, qui réussit à imposer progressivement une solution régionale aux conflits centraméricains : 1980, 1983, 1985, 1987, 1990.

1981, année de la prise de fonctions de R. Reagan à la Maison blanche, est le début de la recherche d'internationalisation. Il accule le régime sandiniste, en décrétant l'embargo commercial et en soutenant massivement la Contra, à une dépendance toujours plus grande du bloc soviétique. Sa constance dans cette volonté d'internationalisation sera exemplaire. En 1984, alors que le Congrès nouvellement élu est à majorité démocrate et risque d'être moins docile que le précédent, il affirme dans un discours à la nation: 
"l'Amérique centrale, c'est l'Amérique. Elle est à nos portes. Elle est devenue le lieu d'une tentative audacieuse de l'Union Soviétique, de Cuba et du Nicaragua d'instaurer par la force le communisme dans l'hémisphère" ${ }^{14}$. L'URSS, pourtant, refuse de se laisser entraîner à une confrontation ouverte avec les USA à propos d'une région où elle sait n'avoir aucune possibilité de s'implanter ${ }^{15}$. Tout au plus cherche-t-elle à éviter la défaite du régime sandiniste, afin de conserver cette monnaie d'échange pour le jour où un éventuel règlement global des affrontements périphériques Est-Ouest interviendrait.

Cette volonté d'internationalisation inquiète beaucoup les Etats voisins, qui redoutent un embrasement général de la région. Pour eux, le risque est sérieux d'une intervention militaire nord-américaine au Nicaragua, qui aurait des effets dévastateurs non seulement dans les Etats d'Amérique centrale, mais aussi dans les Etats limitrophes. Ils sont par ailleurs convaincus de l'aspect contre-productif de la politique des Etats-Unis. Pour eux, il faut empêcher au Nicaragua la réitération du processus cubain, à savoir l'abandon du pluralisme politique et l'arrimage au bloc de l'Est après que les Etats-Unis aient échoué dans leur stratégie de renverser le régime.

La seconde étape débute en 1983, avec la constitution du "groupe Contadora" (Colombie, Mexique, Venezuela, Panama), qui engage une compétition pour imposer une lecture régionale des crises nicaraguayenne et salvadorienne. Ce groupe s'est créé de manière symbolique dans la petite île où avaient été signés les accords CarterTorrijos en 1977. Pendant plus de quatre années, de janvier 1983 à août 1987, il déploie une activité diplomatique très intense, présentant successivement plusieurs plans de paix aux présidents centraméricains, répétant inlassablement qu'une issue négociée aux conflits est possible, qu'il faut la trouver, que leur origine n'est pas à rechercher dans l'affrontement entre les deux blocs mais plus prosaïquement dans l'archaïsme et l'injustice des structures sociales des pays concernés.

Qu'il s'agisse de l'origine des tensions ou des solutions à leurs apporter, le groupe Contadora défendait donc des positions aux antipodes de celles des Etats-Unis. De surcroît, il réussissait à obtenir de nombreux appuis internationaux: celui des ministres des affaires étrangères de la CEE, du Parlement européen, de l'Eglise Catholique, des Eglises protestantes, des membres éminents du Congrès américain, du secrétaire général de l'OEA, celui de l'ONU, et bien d'autres. Ce faisant, il est parvenu à mobiliser l'opinion publique mondiale en faveur de sa démarche, ce qui s'est révélé d'une importance non négligeable ${ }^{16}$.

On n'a pas assez souligné l'importance de l'action de ce groupe ${ }^{17}$, qui a sans nul doute affaibli le clan des faucons à l'intérieur du Pentagone, et a peut-être empêché une intervention militaire nord américaine au Nicaragua. Mais l'originalité de la démarche réside avant tout dans la rupture qu'elle opère dans la longue et complexe histoire des relations entre les Etats-Unis et l'Amérique Latine. Celle-ci était jusqu'alors faite soit de relations bilatérales asymétriques, soit d'un multilatéralisme dominé par les USA. Le passé de l'OEA (Organisation des Etats Américains), ce "ministère des colonies" de Washington, comme l'a un jour appelé Fidel Castro, était là pour l'attester. Cette organisation n'avait jamais réussi à régler quelque problème que ce soit hors de la volonté des Etats-Unis. Avec Contadora, pour la première fois, des Etats d'Amérique latine s'unissaient pour définir une politique et une diplomatie régionales distinctes de celles de Washington. Auparavant on approuvait ou réprouvait, éventuellement dans les enceintes internationales, la politique nord américaine. Mais la concertation sur des 
sujets intéressant les Etats-Unis était impensable hors leur participation, et la réalisation d'une politique autonome eut été de toute manière impossible. C'est parce que les Etats-Unis se voulaient désormais les champions de la démocratie qu'ils ne pouvaient s'opposer ouvertement à une diplomatie élaborée au nom même de cette démocratie. Ils tenteront maintes fois d'influer l'attitude de tel ou tel Etat au sein du groupe, en particulier en usant de savantes mesures de rétorsions commerciales apparemment sans lien avec ses prises de position sur les conflits centraméricains. Cette tactique freina peut-être l'action de Contadora, mais elle ne put l'empêcher de se développer. On est donc bien là en présence d'un fait majeur dans l'histoire des relations interaméricaines.

En 1985, le groupe Contadora est rejoint par quatre pays qui se constituent en "groupe d'appui". Le Pérou, l'Argentine, le Brésil et l'Uruguay s'engagent résolument en faveur de la recherche des solutions négociées. Ces trois derniers pays, rappelons-le, viennent de recouvrer la démocratie avec la bénédiction de Washington. Il convient ici de noter que ces présidents nouvellement élus, à peine sortis de dictatures, tiennent à se démarquer des Etats-Unis et à apporter leur soutien à ce qui apparaît comme une solution latino-américaine. L'habitude de se concerter se prend et, progressivement, ce "groupe des huit", nous le verrons, va s'élargir et étendre son champ de réflexion audelà des problèmes centraméricains.

En 1987 enfin, les cinq pays d'Amérique Centrale (Costa Rica, Guatemala, Honduras, Nicaragua, El Salvador) adoptent, malgré l'opposition affichée de R. Reagan, le plan de paix proposé par le président du Costa Rica, Oscar Arias. Ce plan présentait deux caractéristiques tout à fait originales. D'une part, il liait étroitement les notions de pacification et de démocratisation, affirmant que les deux processus étaient inséparables. L'arrêt des combats et des soutiens externes aux mouvements armés devait s'accompagner d'avancées significatives de la démocratie dans chacun des cinq pays. C'était donc reconnaître que celle-ci devait progresser, y compris dans les pays qui ne connaissent pas de conflits armés mais qui, compte tenu de leur situation géographique, en subissaient les contre-coups.

D'autre part un contrôle régional, garanti par des observateurs extérieurs, devait vérifier les progrès parallèles des deux processus. Des "Commissions de vérification et de contrôle" furent mises en place, comportant pour chaque pays des représentants des quatre autres, assistés de représentants d'organisations internationales et d'observateurs qualifiés. On acceptait donc que des instances internationales contrôlent des processus internes à chaque pays ${ }^{18}$.

L'écho fut immense, amplifié par l'attribution du prix Nobel de la paix à son auteur. Le Congrès des Etats-Unis le soutint avec détermination et refusa désormais de voter les crédits militaires demandés par Ronald Reagan pour la Contra, limitant son aide aux seuls crédits humanitaires. On connaît la suite. Cessez-le-feu, puis paix au Nicaragua en 1989 et 1990, cessez-le-feu, puis paix au Salvador en 1991 et 1992.

La décennie quatre-vingt a donc permis, pour la première fois dans l'histoire du souscontinent, à une diplomatie latino-américaine d'affirmer son autonomie, ceci en partie grâce à la nouvelle doctrine nord américaine de soutien à la démocratie. C'est cette même doctrine qui va être invoquée pour justifier l'opération "Just Cause" à Panama.

Ce pays, on l'a vu, avait initié avec les traités de 1977 un nouveau type de relations interaméricaines. C'est lui qui à nouveau clôt le cycle. Le régime dictatorial du général Noriega est renversé par une intervention armée des USA en décembre 1989. Cette 
intervention, n'est cependant pas tout à fait dans le droit fil de celles du passé. Certes, ici comme dans la longue tradition de ces débarquements de "marines" dans le sous continent, il s'agissait de défendre des intérêts ou des ressortissants nord-américains. Mais la cause première invoquée était la démocratie. Chasser un dictateur qui refusait de reconnaitre le verdict des urnes, et accessoirement le traduire devant la justice américaine qu'il l'avait inculpé pour trafic de drogue ${ }^{19}$.

Cet appel au respect du droit et des procédures démocratiques pour justifier l'invasion d'un pays par un pays tiers était significative du nouvel ordre mondial en train de s'édifier. L'URSS et la communauté internationale protestèrent mollement, tandis que les Etats-Unis faisaient savoir à Gorbatchev qu'ils comprendraient qu'il décidât de recourir à la force pour chasser un autre dictateur, Ceaucescu, qui s'accrochait au pouvoir en Roumanie. Seuls quelques pays d'Amérique Latine, au premier rang desquels le Mexique, manifestèrent leur inquiétude. La pacification des rapports EstOuest signifiait-elle pour eux la perte de cette marge d'autonomie conquise, grâce à l'Amérique Centrale, durant les années quatre-vingts?

Jusqu'alors, sur la scène internationale, l'Amérique n'existait que par ses acteurs étatiques : les Etats-Unis, Cuba, le Mexique, l'Argentine, quelques autres à l'occasion de crises majeures. La plupart des Etats d'Amérique Latine n'avait d'ailleurs jamais manifesté la moindre volonté d'existence sur cette scène, se contentant de relations inter-américaines qui étaient avant tout ordonnées par les Etats-Unis à travers les organisations ou traités régionaux (OEA, TIAR, ALADI, etc.). De nouveaux acteurs subrégionaux, nous l'avons vu, ont surgi à l'occasion de la crise centraméricaine: Contadora, "groupe d'appui", groupe des cinq pays d'Amérique centrale.

Ces acteurs s'étaient constitués sur un objectif unique à savoir résoudre régionalement les conflits de la zone. Il est tout à fait notable qu'ils aient perduré, sous d'autres formes ("groupe des huit", "groupe des trois", "groupe de Rio", etc.) après la fin de ceux-ci et donc l'accomplissement de leur objet. Ils interviennent aujourd'hui en tant que tels, hors de toute existence juridique (aucun traité ne définit ces "groupes"). Le "groupe des trois (Mexique, Colombie, Venezuela) a ainsi défini une politique énergétique commune vis-à-vis de l'Amérique centrale, le "groupe de Rio" (treize pays aujourd'hui) signe des accords avec la CEE, envoie des missions exploratoires en Europe orientale, les réunions bisannuelles des chefs d'Etat centraméricains continuent ${ }^{20}$.

La scène inter-américaine est profondément modifiée par cette nouvelle donne. Désormais, de nombreux acteurs pluri-étatiques américains interviennent dans le champ international, indépendamment des Etats-Unis. Ceci n'a été possible qu'en raison de la disparition de la tension bipolaire. On est ici en présence d'un mécanisme singulier. C'est cette tension sur une crise régionale qui a favorisé l'émergence d'acteurs qui, sans elle, ne se seraient probablement jamais constitués; c'est la disparition de cette tension qui permet leur pérennisation après transformation.

L'écroulement du modèle soviétique au moment de l'arrêt des combats au Nicaragua a enfin eu des conséquences majeures en ce qui concerne le mode de résolution des conflits internes. Tout d'abord, la procédure du dialogue, éventuellement sous les auspices d'observateurs extérieurs, s'est généralisée : les mouvements de guérilla du Salvador, de Colombie, du Guatemala, ont négocié ou sont en train de négocier leur retour sur la scène politique de la démocratie. En second lieu, l'appel à des observateurs étrangers a systématiquement été utilisé pour contrôler les processus politiques les plus controversés (élections au Nicaragua, au Salvador, au Chili, au Pérou...). C'est 
finalement la reconnaissance de la validité du recours à l'aide externe pour éviter le durcissement ou l'émergence de crises internes ${ }^{21}$

Enfin, plusieurs regroupements économiques régionaux (MercoSur, Pacte andin, Accord de libre échange nord américain, Marché commun centraméricain), ont surgi ou ont été revitalisés au début des années quatre-vingt-dix. Nombre de tensions frontalières très anciennes (Guatemala-Belize, Argentine-Brésil, Venezuela-Colombie, accès à la mer de la Bolivie proposé par le Pérou) ont été résolues ou se sont apaisées. Ces regroupements ont pour corollaire une systématisation des rencontres informelles de chefs d'Etat, au cours desquelles s'ébauchent des solutions aux conflits existants ou potentiels (comme le devenir du régime cubain). C'est ainsi qu'à l'initiative du Mexique le principe a été adopté en 1991 d'un sommet annuel de tous les chefs d'Etat ibéroaméricains. 1992 se déroula en Espagne, 1993 sera au Brésil.

Tout se passe finalement en Amérique Latine de manière rigoureusement inverse aux processus d'Europe orientale. Les nouvelles donnes du système international n'ont pas eu ici pour effet d'exacerber les conflits, mais au contraire de les atténuer. Ne doit-on pas y voir là aussi un effet de cette décennie de crises centraméricaines, des modalités de leur déroulement et des procédures inventées pour les résoudre?

Nombre d'observateurs soutiennent que le nouvel ordre mondial aura pour effet de resserrer les liens traditionnellement asymétriques entre les deux Amériques. L'analyse économique inciterait d'ailleurs à conclure à une dépendance accrue de l'Amérique Latine vis-à-vis des Etats-Unis, avec l'objectif nord américain d'une généralisation du libre échange à l'ensemble du continent. Cette conclusion peut de plus être confortée par des considérations plus politiques, à savoir l'importance que revêt désormais le problème de la lutte contre la drogue pour Washington. Il lui faut en effet resserrer ses liens politiques et militaires avec les pays producteurs ou avec ceux, comme dans le cas centraméricain, dont le territoire sert de transit aux envois de cocaïne en provenance des pays andins. De quelle marge d'autonomie disposeront alors l'Amérique Centrale et l'Amérique du Sud, si l'Europe se détourne au profit de sa partie orientale et si les EtatsUnis mesurent leur aide économique au degré de la "coopération" locale à leur politique de lutte contre la drogue?

Paradoxalement, l'ordre mondial relâché22 et la diplomatie de défense de la démocratie pourraient aussi favoriser le maintien de cet espace d'autonomie conquis dans la période écoulée à l'occasion des conflits centraméricains. A partir du moment en effet, et la gestion internationale de la crise du golfe persique l'a amplement démontré, où ce nouvel ordre cherche à se fonder sur le droit international et l'éthique, à partir du moment où les Etats-Unis se posent en champions du respect de ce droit, il leur devient impossible de ne pas respecter eux-mêmes les politiques mises en oeuvre par des gouvernements démocratiquement élus en Amérique Latine. Ceux-ci, et singulièrement ceux des petits pays d'Amérique Centrale, sauront-ils, et surtout pourront-ils, en dépit de l'immensité des problèmes économiques auxquels ils ont à faire face, saisir l'occasion historique qui leur est ainsi offerte de devenir des nations à part entière? 


\section{NOTES}

1. Pour une vision d'ensemble de la région et de ses problèmes, à la fois dans leur dimension historique et durant la décennie 1980, voir l'excellent ouvrage d'Alain Rouquié, Guerres et paix en Amérique centrale, Paris, Seuil, 1992. Voir aussi l'article publié par Olivier Dabène, "Amérique centrale : de la fin de la crise régionale à la poursuite des crises locales" in Cultures \& Conflits, n 1, L'Harmattan, hiver 1990, pp 24-37.

2. Ainsi, en janvier 1990, un administrateur panaméen devait être nommé à la tête du canal. Or en décembre 1989 eut lieu une intervention militaire nord-américaine pour enlever le général Noriega. Ce dernier avait un mois auparavant désigné pour cette fonction un panaméen connu pour son anti-américanisme....

3. Ambrose (Stephen E.), Rise to Globalism, American Foreign Policy since 1938, New York, Penguin Books, 1985 (4 ed. rev.), p. 309.

4. Cf. Rouquié (Alain), op. cit., pp. 178-191.

5. Cf. sur ce point Dabène (Olivier), "L'assistance américaine à l'Amérique centrale (1979-1989)", Problèmes d'Amérique latine, n 91, $1^{\circ}$ trimestre 1989, 115-137.

6. Le Costa Rica a aboli l'armée en 1949. Ce furent donc les forces de sécurité intérieure qui furent l'objet du processus de militarisation durant le mandat du président Monge (1982-1986), provoquant une grave crise de la cohésion nationale. C'est d'ailleurs sur ce terreau que Oscar Arias bâtit sa victoire en 1986. Cf. Muñoz Guillen (Mercedes) et Romero (Rodia), "El dilemno del estado costaricense : segiridad nacional o soberania", in Kruijt (Dirk) et Torres-Rivas (Edelberto), America latina : militares y sociedad, tome 2, San José de Costa Rica, FLACSO, 1991, p.165 sq.

7. Dans le même temps, le Groupe Contadora travaillait avat tout l'opinion publique mondiale (cf. infra). Les Etats-Unis retiendront la leçon lors leur intervention à Panama et surtout au Koweit. Avant, pendant et après ces interventions le souci de s'allier cette opinion fut l'objet d'une stratégie particulièrement élaborée.

8. Une abondante littérature existe sur la "guerre de basse intensité". Cf. par exemple Barry (Deborah), "'Los conflictos de baja intensidad : el caso de Centroamérica", in Vergara Meneses (Raúl) et alii, Centroamérica : la guerra de baja intensidad, San José de Costa Rica, DEI, 1987, 27-50.

9. Tulchin (Joseph) et Walter (Knut), "Nicaragua : The Limits of Intervention", in Lowenthal (Abraham F.), Exporting Democracy : The United States and Latin America, Baltimore and London, The Johns Hopkins University Press, 1991, p.134. Dans le même ouvrage, voir aussi le chapitre de Laurence Whitehead, "The Imposition of Democracy", en particulier, pour le Nicaragua, ce qu'il qualifie de "promotion de la démocratie par intimidation" (p.249 sq.)

10. On trouvera une bonne illustration de la position nord américaine dans un ouvrage couronné par le prix Pulitzer, Christian (S.), Nicaragua, Revolution in the Family, New York, Random House, 1985, pp.297-311.

11. On retrouve d'ailleurs un situation similaire au Chili, où le président Aylwin n'a pas tenté de modifier une constitution qui garantit au général Pinochet de demeurer chef des forces armées jusqu'en 1997.

12. On en trouvera une bonne présentation dans l'ouvrage coordonné par Rouquié (Alain), Les forces politiques en Amérique centrale, Paris, Karthala, 1991. 
13. Voir sur ce point notre chapitre "A qoui sert de voter en Amérique latine ?", in Couffignal (G.), (dir.), Réinventer la démocratie : le défi latino-américain, Paris, Presses de la FNSP, 1992, 19-48.

14. Cité par Sohr (Raül) in Centroamérica en guerra, Mexico, Alianza Editorial Mexicana, 1988, p20.

15. Nicolas Miller a ainsi montré , en étudiant de manière approfondie les cas du Chili, de la Grenade et du Nicaragua, qu'après la crise cubaine l'Union Soviétique n'a à aucun moment cherché à exploiter l'arrivée au pouvoir de formations de gauche en Amérique Latine, les incitant au contraire à normaliser leurs relations avec les Etats-Unis : Miller (Nicolas), Soviet Relations whith Latin America, Cambridge, Cambridge University Press, 1989.

16. Cf. sur ce point notre étude Couffignal (Georges), "Amérique centrale, démocratie et relations internationales", in Quel avenir pour la démocratie en Amérique centrale, (ouvrage collectif), Toulouse, Editions du CNRS, 1989, 73-83. Les Etats-Unis retiendront cette importance de l'opinion publique mondiale lorsqu'ils prendront la tête de l'intervention militaire au Koweit...

17. On se reportera avec profit à une très bonne thèse de science politique qui y est en grande partie consacré : Ramirez (Socorro), "Les marges d'autonomie de la politique extérieure colombienne", mimeo, Paris I-Sorbonne, 1992, 410p.

18. On trouvera le texte de l'accord dans le dossier constitué par Marie-Chantal Barre, "Amérique centrale : une dynamique de la paix", Problèmes politiques et sociaux, 593, oct. 1988), pp.26-32.

19. Cf. notre étude "L'intervention de décembre 1989 à Panama" 'Big Stick', bulletin de vote et 'Mare Nostrum'", Herodote, $\mathrm{n}^{\circ} 57,2^{\circ}$ trimestre 1990), pp.76-86.

20. voir sur cepoint notre étude, "Mono-idéologique, complexe et relâché : le système interaméricain après la guerre froide", in Laïdi (Zaki), (dir), L'ordre mondial relâché, Paris, Presses de la FNSP, 1992, 209-236.

21. C'est d'ailleurs à cette même époque (décembre 1989) que les notions de droit ou devoir d'ingérence, qui trouvent leur pleine reconnaissance avec l'intervention "onusienne" en Somalie, ont pour la première fois été formulés (à propos de Panama et de la Roumanie)

22. Selon la formule proposée par Zaki Laïdi "in "Sens et puissance dans le système international", Laïdi (Zaki), (dir), L'ordre mondial relâché, op. cit., pp.18-46.

\section{RÉSUMÉS}

Contrairement à d'autres sphères régionales on semble assisté en Amérique Centrale à un apaisement des conflits. Tout se passe comme si les nouvelles donnes du système international avaient en définitive eu un effet "positif ", à savoir celui de modérer l'intensité des conflits dans la région ou même d'y mettre fin. On ne saurait certes pas imputer cette accalmie à la seule disparition du bloc soviétique, les acteurs régionaux et sub-régionaux, en premier lieu les USA ont "contribué" à cette nouvelle situation. 
In contrast to other regions, conflicts in Central America seem to be on the wane. It looks as if the new international system ultimately had a " positive " effect, i.e., in abating the intensity of conflicts or even in putting an end to them. This lull cannot be exclusively ascribed to the disappearance of the Soviet block ; it is largely due to the efforts of the regional and sub-regional powers, mainly the USA.

INDEX

Index géographique : Amérique centrale

Mots-clés : crises politiques, régionalisme et régionalisation

Index chronologique : 1980 - 1990

Thèmes : Contras 